\title{
ESTUDIANTES UNIVERSITARIOS EN SITUACIÓN DE EXAMEN DE ÚLTIMA OPORTUNIDAD DE INGLÉS I \\ UNIVERSITY STUDENTS AND THE LAST ENGLISH I EXAMINATION
}

\author{
Volumen 15, Número 1 \\ Enero - Abril \\ pp. 1-20
}

Este número se publicó el $1^{\circ}$ de enero de 2015

DOI: dx.doi.org/10.15517/aie.v15i1.17589

Claudia Isabel Marín Sánchez

Emily LibradoTorres

María Eugenia Alarcón Aguirre

Revista indizada en REDALYC, $\underline{\text { SCIELO }}$

Revista distribuida en las bases de datos:

CATÁLOGO DE LATINDEX, IRESIE, CLASE, DIALNET, DOAJ, E-REVIST@S, SHERPA/ROMEO, QUALIS, MIAR

Revista registrada en los directorios:

ULRICH'S, REDIE, RINACE, OEI, MAESTROTECA, PREAL, CLACSO 


\title{
ESTUDIANTES UNIVERSITARIOS EN SITUACIÓN DE EXAMEN DE ÚLTIMA OPORTUNIDAD DE INGLÉS I \\ UNIVERSITY STUDENTS AND THE LAST ENGLISH I EXAMINATION
}

\author{
Claudia Isabel Marín Sánchez ${ }^{1}$ \\ Emily LibradoTorres ${ }^{2}$ \\ María Eugenia Alarcón Aguirre ${ }^{3}$
}

Resumen: Este artículo es un estudio cualitativo exploratorio sobre las causas que ocasionaron que estudiantes de la Universidad Veracruzana en México presentaran el examen de última oportunidad del curso de Inglés I. Se identificaron las causas que ocasionaron que presentaran este examen. Se entrevistaron estudiantes, personal académico del Centro de idiomas y de los Centros de Autoacceso. Los resultados demostraron falta de interés por el curso y desconocimiento del aprendizaje autónomo. Se propone capacitación continua a tutores y tutoras sobre las modalidades para cursar inglés, cursos de aprender a aprender y la creación de una red de comunicación para apoyar estudiantes en riesgo de reprobar esta experiencia educativa.

Palabras clave: INGLÉS, AUTONOMÍA, RESPONSABILIDAD, COMPORTAMIENTO ESTUDIANTIL, EDUCACIÓN SUPERIOR, MÉXICO.

Abstract: This research project is exploratory, qualitative and identifies the factors that lead students to an English final test called "the last-chance test". It was conducted at Universidad Veracruzana in Mexico. The study aimed to identify reasons why students have to make this exam. Participants were students, teachers and assessors from the Self-Access Centers. Results showed that the main reasons were lack of interest and lack of training in autonomous learning. We propose training for counselors, learning to learn workshops and an effective communication system to support students at risk of failing.

Key words: ENGLISH, AUTONOMY, RESPONSIBILITY, STUDENT BEHAVIOR, HIGHER EDUCATION, MÉXICO.

\footnotetext{
1 Docente del Centro de Idiomas Coatzacoalcos de la Universidad Veracruzana, México. Dirección electrónica: cmarin@uv.mx

2 Docente del centro de idiomas Coatzacoalcos de la Universidad Veracruzana, México. Dirección electrónica: elibrado@uv.mx

3 Docente del centro de idiomas Coatzacoalcos de la Universidad Veracruzana, México. Dirección electrónica: eualarcon@uv.mx
}

Artículo recibido: 5 de mayo, 2014

Enviado a corrección: 29 de julio, 2014

Aprobado: $1^{\circ}$ de diciembre, 2014 


\section{Introducción}

A partir del siglo XX, la educación superior en América Latina y el Caribe ha tenido tres reformas que hoy en día permiten percibir la significativa evolución que ésta ha tenido en términos de democratización y expansión (Rama 2006, pp. 14-17), inclusión de grupos considerados minoría (personas con capacidades diferentes, de origen indígena, migrantes, etc.) así como los grandes retos que debe enfrentar gracias a la internacionalización y el uso masivo de las nuevas tecnologías de comunicación. No obstante, los índices de reprobación en estudiantes de universidad siguen siendo un punto neurálgico que aún requiere atención. De acuerdo con el censo de población y vivienda realizado en el 2010 en México por el Instituto Nacional de Estadística y Geografía (INEGI), el grado promedio de escolaridad equivale a tercero de secundaria; esto significa que un número reducido de jóvenes logra matricularse en la universidad, pero menor aún es el número de los que la concluyen.

Entre los motivos por los cuales el estudiantado no concluye sus estudios universitarios está el fenómeno de la reprobación. Según Rodríguez (1999, p. 1), reprobación significa "insuficiente rendimiento cuantitativo y/o cualitativo de las potencialidades de un alumno para cubrir los parámetros mínimos establecidos por una institución educativa." Pero, ¿cuáles son las causas de reprobación en estudiantes de universidad? El fenómeno de reprobación en instituciones de educación superior ya ha sido analizado desde diversas perspectivas. Martínez Maldonado, Vivaldo Lima, Navarro Padilla, González de la Fuente y Jerónimo Montes (1998) relacionaron el índice de reprobación en una facultad de la Universidad Autónoma de México con variables de tipo social (el nivel educativo y económico de la familia), individual (hábitos de estudio, motivación, estilo cognoscitivo) e institucional (la infraestructura, el currículo, el proceso de enseñanza-aprendizaje).

En un estudio similar realizado en la Benemérita Universidad Autónoma de Puebla, Cabrera Sánchez, Romano Rodrígue y Valenzuela Ojeda (2006) identificaron que este fenómeno era provocado por tres causas: los estudiantes, los docentes y el plan de estudios. En la Universidad Veracruzana, Aldana Franco, Mora Barradas, Ricaño Herrera, Álvarez Sánchez, López Velázquez, Navarro Piedra, Solórzano Hernández, Ramírez Ramírez, García Araujo, Hernández Flores y Rosas, encontraron que la reprobación en estudiantes de la Facultad de Ingeniería Mecánica Eléctrica se debe a "que no cuentan con antecedentes sólidos en matemáticas" (2012, p. 1) y a la dificultad de adaptarse al modelo educativo de la institución. 
En el caso de la reprobación, particularmente en el área de inglés, las causas suelen ser similares. Un claro ejemplo es el estudio realizado por Reyes (2002) en la Universidad de Quintana Roo, quien enfatizó que la situación económica precaria de algunos y algunas estudiantes los obliga a trabajar al mismo tiempo que estudian; estas condiciones les ocasionan cansancio excesivo y falta de cumplimiento en sus tareas escolares. Otros factores importantes del mismo estudio son los problemas familiares y la falta de hábitos de estudios apropiados. En relación con la materia de inglés, en la Universidad Veracruzana, se realizó un estudio sobre la depresión en estudiantes con rezago en inglés I y II (Luna Martínez, Barradas Alarcón y Balderrama Trápaga, 2012). Sin embargo, las causas de reprobación en estudiantes de inglés en la Universidad Veracruzana es un aspecto que vale la pena estudiar porque los resultados pueden llevar a identificar las causas particulares de este fenómeno para proponer alternativas de solución que puedan ser replicables en contextos similares.

\section{Contexto}

En el año 1999 se implementó el Modelo Educativo Integral y Flexible (MEIF) en la Universidad Veracruzana (UV) en México. Este modelo, de acuerdo con Beltrán (2005, p. 4), busca "educar integralmente al estudiante [...], y establecer como obligatorio el aprendizaje de habilidades de comunicación y de autoaprendizaje", entre otros aspectos.

La experiencia educativa Inglés I es un curso-taller que pertenece al Área de Formación Básica General (AFBG) del MEIF. El AFBG está compuesta por 5 experiencias educativas (EEs): Computación Básica, Taller de Lectura y Redacción, Habilidades del Pensamiento Crítico y Creativo, Inglés I e Inglés II; estas experiencias son de carácter obligatorio para todos los programas educativos (PEs) de la UV y deben ser acreditadas antes de cubrir el $50 \%$ de los créditos totales del Pes correspondiente. De no hacerlo, el estudiante o la estudiante no puede inscribirse al semestre próximo para continuar cursando otras experiencias educativas (Yerena, 2000, pp. 2-3).

Las experiencias educativas (EEs) Inglés I y II son cursadas en la modalidad de taller. Si un o una estudiante lo reprueba, deberá cursarlo por segunda vez. Estas experiencias pueden ser cursadas en tres modalidades: presencial, autónoma y abierta. En la modalidad presencial, las jóvenes y los jóvenes asisten a clases con su docente y un horario establecido. Así mismo, se busca desarrollar la competencia comunicativa y la autonomía para que puedan utilizar el idioma inglés fuera del salón de clases (Brown, 2007, pp. 79-81). 
Este curso consta de seis horas a la semana. En la modalidad autónoma, se debe asistir al Centro de Autoacceso (CAA) sin un horario fijo dónde se cursa la EE de manera autónoma con asesoría del personal académico. El sistema abierto ofrece cursos presenciales de estas EEs que consta de dos horas de clases únicamente los sábados y el resto de las sesiones se cubren con asesorías y actividades en línea y/o a distancia.

El alumnado que ya tiene conocimientos del idioma inglés pueden acreditar estas EEs por medio del examen de competencias en el que se evalúan las cuatro habilidades (expresión oral y escrita, comprensión auditiva y de lectura). También pueden acreditarlas por medio de exámenes de certificación (EXAVER de la Universidad Veracruzana (UV), PET, KET, First Certificate y TOEFL) y los cursos que se imparten en los Centros de Idiomas y en el Departamento de Lenguas Extranjeras de la UV a través de la transferencia de créditos. A partir del año 2008, el estudiantado tiene derecho a cursar cada EE dos veces en cualquier modalidad. Sin embargo, algunas personas cursan y reprueban estas dos oportunidades.

En consecuencia, deben solicitar y presentar un examen llamado de Ultima Oportunidad (UO). El estatuto de los Alumnos 2008 establece que "Son causas de baja definitiva: III. Reprobar un examen de última oportunidad en los niveles que aplique;" (Título IV, capítulo I, Sección Segunda, artículo 40 fracción III del Estatuto de los Alumnos, 2008). Como su nombre lo indica, este representa la última oportunidad para acreditar la EE a través de un examen global. Si no lo acredita, se le da de baja definitiva de su Programa Educativo (PE) sin importar el número de créditos que haya cursado. La reprobación de un examen de última oportunidad afecta no sólo al estudiantado quienes son dados de baja definitiva de su programa educativo, sino también a las Facultades a las que pertenecen ya que el índice de deserción se incrementa afectando de manera negativa sus indicadores de eficiencia terminal. Como lo muestra la tabla 1, durante el periodo agosto- diciembre 2010 de 963 estudiantes que cursaron en inglés I en la Región Coatzacoalcos-Minatitlán, el 33\% no acreditó la EE. Mientras que en el periodo enero - julio 2011, de 361 estudiantes, el 50 \% no acreditó. 
Tabla 1

Comparación del resultado final de estudiantes inscritos en los periodos agosto diciembre 2010 y enero - julio 2011

\begin{tabular}{|c|c|c|}
\hline Periodo & $\begin{array}{c}\text { Estudiantes inscritos en } \\
\text { inglés I }\end{array}$ & $\begin{array}{c}\text { Estudiantes que no acreditaron } \\
\text { inglés I }\end{array}$ \\
\hline Agosto - diciembre 2010 & 963 & 324 \\
\hline Enero - julio 2011 & 361 & 181 \\
\hline
\end{tabular}

Fuente: Elaboración propia con base en las Actas de calificaciones proporcionadas por el Centro de Idiomas Coatzacoalcos y Facultades de la Región (2012)

Es importante mencionar que del $33 \%$ del estudiantado que no acreditó en el primer periodo mencionado, el $13 \%$ no asistió al examen final y el $9 \%$ no tuvo derecho a presentarlo. Mientras que en el periodo de enero - julio 2011 del 50 \% que no acreditó la EE, el $15 \%$ no presentó y el $23 \%$ no tuvo derecho a ser evaluado. En el gráfico 1, se visualizan estos datos así como las modalidades cursadas.

\section{Gráfico 1}

Resultado obtenido por estudiantes de inglés I y la Modalidad cursada en los periodos agosto -diciembre 2010 y enero-julio 2012

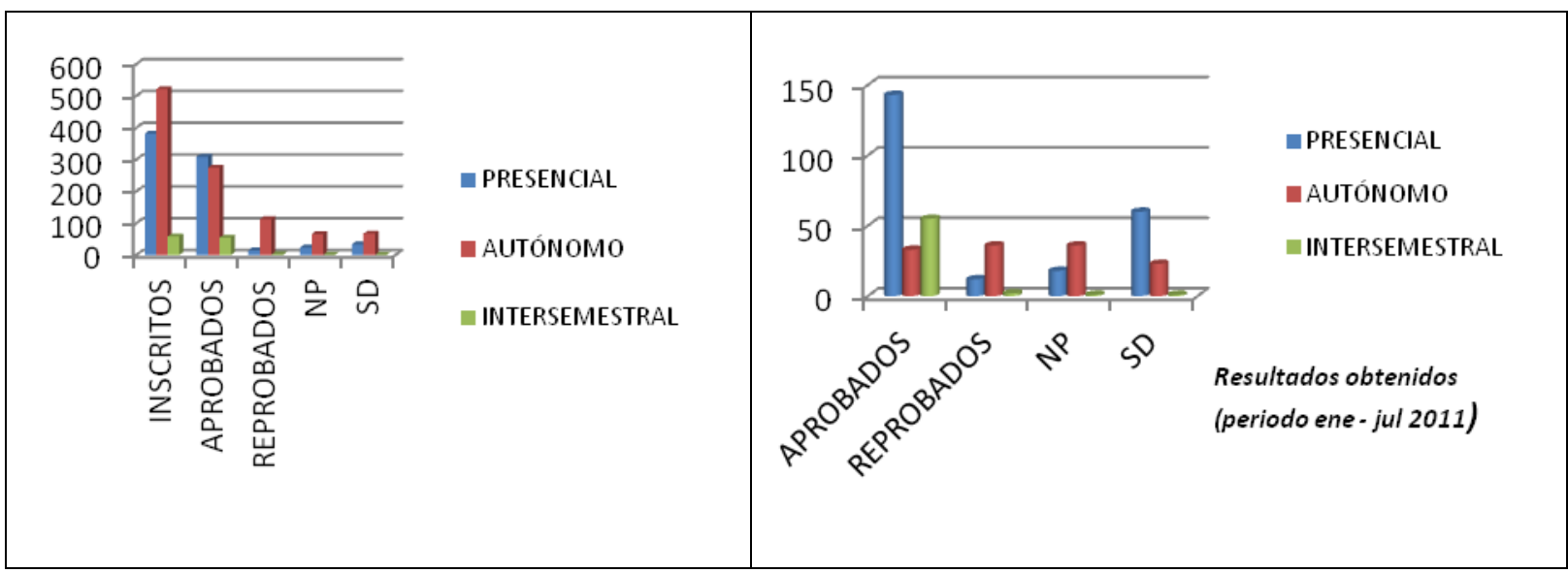

Fuente: Elaboración propia (2013)

\section{Metodología}

\subsection{Objetivos}

Los objetivos que guiaron esta investigación fueron:

1. Identificar los criterios que utiliza la comundad universitaria para elegir la modalidad bajo la cual cursan la experiencia educativa Inglés I MEIF. 
2. Analizar los factores que afectan el desempeño de la población estudiantil y que provocan que reprueben considerando el punto de vista estudiantil y la perspectiva académica.

3. Ofrecer posibles alternativas de soluciones que podrían ayudar a disminuir el número de estudiantes que presentan el examen de última oportunidad.

\subsection{Participantes}

El estudio se llevó a cabo con tres grupos de participantes: a) 17 estudiantes que habían presentado el examen de UO identificados por medio de una lista proporcionada por la Coordinación del Centro de Idiomas; b) 2 profesores y 4 profesoras y c) 4 asesoras.

\subsection{Instrumentos}

a) Actas de exámenes ordinarios de los períodos Agosto-Diciembre 2010 y Enero-Julio 2011 solicitadas a Facultades y al Centro de Idiomas Regional.

b) Guía de preguntas para cada grupo de participantes (ver Anexo 1, 2 y 3).

\subsection{Procedimiento}

Para hacer el análisis de los datos se solicitaron las actas de exámenes ordinarios de los períodos Agosto-Diciembre 2010 y Enero-Julio 2011 a las diferentes Facultades y al Centro de Idiomas regional. Posteriormente, se contabilizó el número de estudiantes de acuerdo con el resultado obtenido (aprobados, reprobados, no se presentó, sin derecho), el programa educativo al que pertenecen y la modalidad en que cursaron la EE. Se diseñó una guía de preguntas para cada grupo de participantes con el objetivo de tener una visión real y auténtica de cómo los entrevistados ven esta problemática. Las preguntas guía buscaban propiciar la auto reflexión y motivarlos a compartir sus puntos de vista.

La recolección y el análisis de los datos se realizaron según el procedimiento de Creswell (1994) y Hernández, Fernández y Baptista (2010). Para la recolección de datos, se entrevistaron a estudiantes que presentaron el examen de UO de inglés I en junio de 2011 y en junio de 2012. A continuación, se realizaron las entrevistas a profesores, profesoras y asesoras siguiendo el mismo procedimiento. Cabe mencionar que la muestra fue de conveniencia porque no toda la población de estudiantes que presentaron este examen accedieron a participar y no todo el personal académico fue entrevistado. Las entrevistas fueron grabadas, transcritas y analizadas para la identificación de categorías. Por último, se 
realizó la triangulación de la información obtenida de los tres grupos de participantes para obtener datos representativos que permitieran cumplir con los objetivos de este estudio.

\section{Análisis y discusión}

Con el análisis de las entrevistas a estudiantes y personal académico se identificaron las siguientes categorías:

\subsection{Semestre en el que cursaron la primera y segunda oportunidad}

Se consideró importante identificar este dato con las personas entrevistadas porque se ha observado que algunas/os jóvenes ya están a más de la mitad de su avance crediticio cuando reprueban y deben abandonar sus estudios por no acreditar la EE de inglés I la cual debieron cursar desde primer semestre:

Tabla 2

Relación de estudiantes y el semestre en que cursaron su primera inscripción a inglés

\begin{tabular}{|c|c|c|c|}
\hline \multicolumn{2}{|c|}{ Primera inscripción } & \multicolumn{2}{|c|}{ Segunda inscripción } \\
\hline Semestre & Número de estudiantes & Semestre & Número de estudiantes \\
\hline Primero & 4 & Segundo & 0 \\
\hline Segundo & 9 & Tercero & 13 \\
\hline Tercero & 1 & Cuarto & 0 \\
\hline Cuarto & 2 & Quinto & 3 \\
\hline Quinto & 1 & Sexto & 1 \\
\hline
\end{tabular}

Fuente: Elaboración propia (2013).

Como se puede observar en la tabla 2, las/os entrevistadas/os realizaron su primera inscripción a la EE de inglés I en cualquier semestre y no necesariamente desde el primer semestre. Se identificó que cierto número de estudiantes hicieron su segunda inscripción inmediatamente después de haber reprobado, mientras que otros dejaron pasar un periodo escolar antes de hacer su segunda inscripción.

\subsection{Información recibida sobre las modalidades}

La información conocida por la población estudiantil acerca de las diferentes modalidades ofrecidas para cursar la EE era muy vaga; es evidente que había mucha confusión. Algunas personas recibieron información aunque no la recordaban bien; otras se inscribieron a la EE porque ya la tenían asignada desde sus Facultades. La población 
estudiantil de nuevo ingreso reportó estudiantes de nuevo ingreso reportaron que desconocían el sistema de trabajar en la Universidad Veracruzana. "Yo no tenía conocimiento, de hecho, todavía me han quedado muchas dudas al respecto. De haber sabido o haber tenido la información necesaria, me hubiese inscrito desde primer semestre", comentó uno de los estudiantes entrevistados. Mientras que una alumna comentó vagamente que les hablaron sobre "las modalidades y las oportunidades".

Desde el punto de vista del personal docente, la información recibida sobre las modalidades es muy escasa, opinaron que sólo se informan de las dos oportunidades que tienen para aprobar la experiencia de Inglés I, y no le dan mucha importancia a la información que reciben en el programa "Conoce tu Universidad"4, desconocen las modalidades existentes y solo se inscriben porque así se les pide en su Facultad. Con respecto a esto, una profesora entrevistada comentó “...bueno, traen poca información porque no todos saben de las oportunidades que tienen para no tomar el curso, los exámenes de competencia, algunos no tienen el conocimiento de que hay esas posibilidades". Otro profesor agregó: "ellos carecen o se les olvida que tienen diversas oportunidades, las pueden presentar en diferentes modos presencial, autónomo, presentar un examen de competencia, presentar EXAVER, movilidad...algunos de ellos si olvidan que tienen estas oportunidades y a veces, tal vez se les va...el adecuado".

Las asesoras entrevistadas coincidieron con el sentir de las demás personas entrevistadas los profesores y estudiantes. Aunque existe el programa Conoce tu Universidad, las asesoras opinaron que la información que se les proporciona a estudiantes de nuevo ingreso no era suficiente. Sobre esto, las asesoras comentaron: "creo que es una gran problema porque la información que ellos saben en cuanto a los tipos de modalidades que existen a veces es nula, a veces ellos ni siquiera saben que pueden hacerla de manera presencial o que tienen la opción de hacerla en el Centro de Idiomas". "Cuando los alumnos ingresan a la universidad se les hace en los primeros días de su ingreso una explicación a grandes rasgos de cómo está programada la carga académica en esos primeros días se les da una idea general sobre lo que es el autoacceso y el trabajo que van hacer de manera autónoma."

\footnotetext{
4 Programa institucional de actividades tales como pláticas, visitas y asesorías que se realiza para los estudiantes de nuevo ingreso durante la primera semana de clases con el objetivo de que se familiaricen con la infraestructura y la forma de trabajo
} 


\subsection{Modalidad en la que cursaron la primera y segunda inscripción a la EE}

Se observó que la población estudiantil cuya primera inscripción fue en la modalidad autónoma (ya sea porque así apareció en el sistema, porque pensaron que era más fácil cursarla de esta manera o porque los horarios de los cursos presenciales no eran accesibles), en la segunda oportunidad, alguna personas optaron por inscribirse en la modalidad presencial tal como puede observarse en el gráfico 2:

\section{Gráfico 2}

Relación de estudiantes y la modalidad cursada en las dos inscripciones

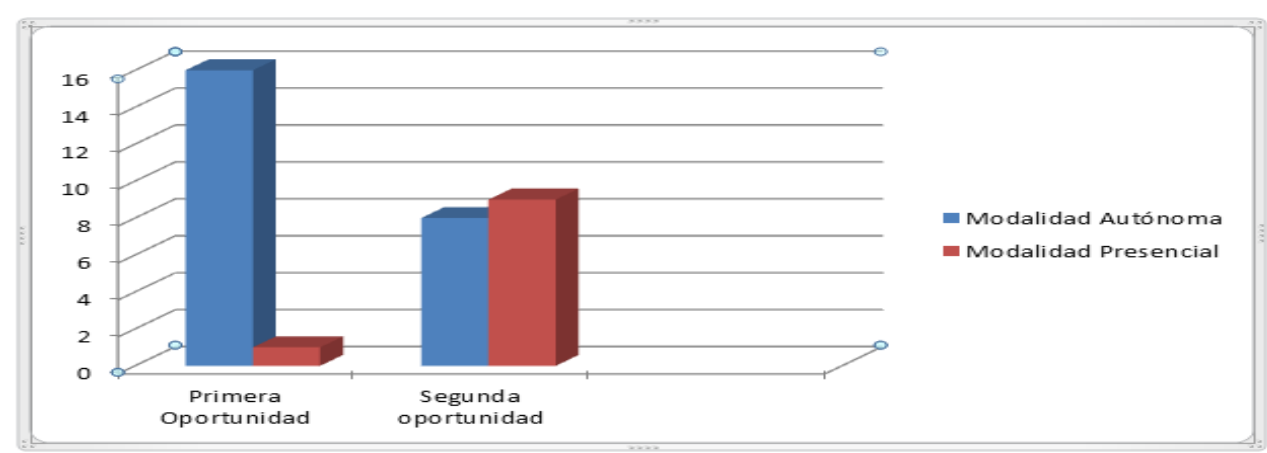

Fuente: Elaboración propia (2013).

\subsection{Razones por las que eligieron la modalidad cursada}

Un grupo de estudiantes eligieron la modalidad presencial porque así les aparecía en el sistema en línea y ya no se interesaron en investigar otras opciones, aquí sus comentarios: "Sí es que a veces abren de.... en clases presenciales, ya no había grupos y por eso me inscribí en autónomo", "porque a nosotros ya nos aparecía como un bloque de materias". Sobre esto, un profesor comentó "la eligen a veces por ignorancia, porque algunos no saben que existe la modalidad autónoma y otros porque es la costumbre, creen que solo existe esa, y pues la inscriben porque aparece en su horario". Por otra parte, las asesoras entrevistadas explicaron que la mayoría de estudiantes inscritos en el CC desconocían el sistema de aprendizaje autónomo. Por lo tanto, al cursar la EE por segunda vez, esta población prefería las clases tradicionales donde se les guía y se les indica las actividades a realizar teniendo así una falsa idea de que la responsabilidad de aprendizaje pertenece al docente.

Sobre este punto, una profesora comentó lo siguiente: "yo creo que nuestros estudiantes carecen todavía de esta práctica de la autonomía o de la independencia de ser un estudiante autónomo y por ello siguen optando por el modo presencial en donde hay un 
maestro". Asimismo señalaron que el desconocimiento de las diferentes modalidades que la universidad ofrece para acreditar la EE ha afectado, particularmente, a estudiantes que la han cursado en la modalidad autónoma.

Las asesoras consideraron que los estudiantes a menudo eligen la modalidad autónoma por razones equivocadas: 1) ésta no cuenta con horario establecido y la asistencia al CAA es flexible, lo cual les hace creer que es más fácil, que el trabajo se realiza únicamente en línea y que no es necesario asistir al CAA. Ellas señalaron que existe un gran desconocimiento del aprendizaje autónomo: "Poquito trabajo, es algo así que puedes hacer desde casa y ni siquiera tienes que venir aquí al autoacceso y así tienes más tiempo para dedicarle a tus materias"; 2) En otros casos, es el personal directivo de la facultad quien decide cuándo y en qué modalidad cursar la EE: “...ellos no tienen ese tipo de información, ellos generalmente llegan y dicen a mí me dijeron que me tenía que inscribir a ingles en el centro de autoacceso,....; 3) La carga académica excesiva lleva a la búsqueda de un horario que no se traslape con las otras EEs: "muchos de los usuarios eligen la modalidad autónoma para cursar su materia porque les permite libertad de tiempo de llevar su estudio de acuerdo a su modo de estudio y se les hace muy cómodo la flexibilidad de horario.

\subsection{Total de EE's cursadas al mismo tiempo que cursaban inglés I}

Como se puede ver en la tabla 3 , en la primera inscripción, 9 estudiantes se matricularon en 7 EE's, mientras que en la segunda inscripción, 8 estudiantes cursaban 6 EE's al mismo tiempo que cursaban inglés. Es necesario mencionar que 2 personas entrevistadas cursaban entre 9 y 10 EE's al mismo tiempo que inglés.

Tabla 3

Relación del número de personas entrevistadas y la cantidad de experiencias educativas que cursaban al mismo tiempo que estaban inscritos en inglés I

\begin{tabular}{|l|c|c|c|c|c|}
\hline \multicolumn{7}{|c|}{ Primera inscripción/ oportunidad } \\
\hline Número de experiencias educativas & 6 & 7 & 8 & 9 & 10 \\
\hline Número de estudiantes & 3 & 9 & 2 & 1 & 1 \\
\hline
\end{tabular}

\begin{tabular}{|l|c|c|c|c|c|}
\hline \multicolumn{7}{|c|}{ Segunda inscripción/oportunidad } \\
\hline Número de experiencias educativas & 6 & 7 & 8 & 9 & 10 \\
\hline Número de estudiantes & 8 & 4 & 2 & 1 & 1 \\
\hline
\end{tabular}

Fuente: Elaboración propia (2013) 


\subsection{Causas de reprobación en ambas inscripciones}

En el análisis de las entrevistas realizadas a estudiantes, se identificaron cuatro principales causas que se presentan a continuación junto con las transcripciones de los comentarios:

Falta de interés. No le daban importancia a la experiencia educativa de inglés ya que preferían cumplir con las EEs del área disciplinar.

Falta de compromiso con la experiencia educativa de inglés I. El grupo de estudiantes mencionó que no cumplieron con las actividades ni con los tiempos en que debían entregarlos, además de que no asistían a clases o al autoacceso.

Entrevistado 3: "No cumplía con los tiempos en que se debían entregar las

actividades, le di más importancia a otras EE”.

Entrevistado 8: "No tenía la responsabilidad de estudiar y no le tomaba importancia".

Entrevistado 6: "No hacía los trabajos, no iba a Autoacceso".

Entrevistado 2: La primera ocasión empecé bien entregando los trabajos en la plataforma igual iba al cadi a [...] pero después se me fue juntando lo de la carrera y fui postergando decía después [...] y en la segunda oportunidad darle prioridad a las otras materias [...] y fue que decidí dejarlo y dije bueno creo que me conviene más hacer un sólo examen y ya así no tengo que estarme preocupando por hacer mis actividades o la libreta o cosas así".

Dificultad para adaptarse al sistema. No entendían cómo era el sistema autónomo y no preguntaron cómo trabajar en él.

Entrevistado 1: "No sabía si había asesores...".

Entrevistado 7: "reprobé porque no hice plataforma no acudí a los clubes de conversación y me hicieron falta como dos o tres bitácoras".

Entrevistado 4: ““creo que fue que no entendía muy bien lo de la plataforma y este a pesar de que se ve muy sencillo entrar de la computadora y hacerlo desde la computadora este no sé ...".

Entrevistado 5: "Es que no le entendía a lo del sistema de cómo es que calificaban de cómo subir mis tareas, cuando tenía que venir, las fechas".

Entrevistado 1: Por la falta de interés como no me gusta no tuve ese hábito de investigar e indagar sobre la materia de igual manera y aparte como fue autónomo se me 
hizo un poquito medio extraño trabajar en línea además utilizaba la mayor parte del tiempo a trabajar".

Trámites administrativos. No haber dado de baja la EE en tiempo y forma ocasionó que apareciera en el sistema como cursada.

Entrevistado 3: "reprobé EXAVER y no me dieron de baja antes de presentar el examen, yo pensé que sí lo habían hecho".

Entrevistado 8: "No me cambié porque no me dieron de baja a tiempo; reprobé. No sabía bien como era la inscripción al Centro de idiomas, habían colas muy largas. La opción que encontré fue cursar la EE autónoma en el CAA. Esta información la obtuve de mis compañeros, nunca pregunté a alguien del $\mathrm{Cl}$ o del CAA porque había que venir en la mañana y no puedo porque trabajo y pensé que en la tarde no me iban a dar información".

Entrevistado 6: "No pude cancelar la inscripción y por el horario llegaba tarde".

Aun cuando el programa de Inglés I incluye, además del examen final, profesores varias actividades al evaluar, algunas/os estudiantes no acreditan. En gran parte esto se debe a la falta de interés, al incumplimiento de las actividades y a la inasistencia a la clase o al CAA. En ocasiones, la inasistencia obedece a factores tales como condiciones climatológicas adversas o compromisos laborales. Como consecuencia, la EE se hace difícil y, poco a poco, el estudiantado se va desanimando hasta que ya no hacen ningún esfuerzo por aprobar. En casos extremos, pierden su derecho a presentar el examen final. Sobre este punto una profesora entrevistada afirmó que: "les cuesta mucho trabajo y además de que no repasan en casa a pesar de que se les exige, que realicen sus tareas y cosas así... este... y también se confían porque piensan que es una materia de relleno". Se han identificado, en algunos casos, estudiantes que llegan con un nivel muy bajo de inglés.

Las asesoras entrevistadas identificaron varias causas de reprobación en la modalidad autónoma; entre ellas, se identificaron la poca o nula asistencia al CAA debido a la excesiva carga de EE's, la incorrecta organización del tiempo y el empleo del mismo en actividades de esparcimiento que deja al grupo de estudiantes con poco tiempo disponible para asistir al CAA y realizar las actividades correspondientes. Otra causa identificada es la inseguridad que provoca trabajar en un sistema autónomo al cual muchas/os estudiantes no están acostumbrados. Debido a que la evaluación es sumativa, el porcentaje final no es suficiente para acreditar.

Las asesoras coincidieron en que el éxito al trabajar en la modalidad autónoma se basa en la organización y la disciplina además de la capacidad de entender que es la/el 
estudiante quien construye su propio aprendizaje. Esto se ve reflejado en los siguientes comentarios:

Asesora 3: "Yo creo que la más importante es la falta de información. Otra muy importante es que el alumno que llega a la universidad no es un alumno autónomo, no está preparado para ser un alumno autónomo, entonces le cuesta mucho hacerse responsable de todo su aprendizaje, le cuesta mucho tener en cuenta las fechas en que debe venir, las fechas en que debe hacer exámenes, todo lo deja a la ultima hora y además nos siguen considerando como un extra".

Asesora 1: "Por la falta de estudio desde los objetivos porque eso es lo que ellos hacen de manera autónoma y todo ese material esta aquí, está en el autoacceso y está en la plataforma. Siento que porque no le están dando la mayor importancia al área de inglés, están ocupados en estudiar sus materias. Manejo del tiempo, la falta de programación el alumno autónomo debe de ser responsable de su propio aprendizaje, debe de ser organizado".

Asesora 2: "creo que reprueban primero porque muchos no están acostumbrado a trabajar por ellos mismos ya sean solitarios o apoyándose en compañeros o en los asesores he en la carga académica que tienen en sus Facultades que les permite poco tiempo libre para venir al centro de autoacceso ya que ellos en el centro de autoacceso vienen a la hora en que disponen de tiempo de sus otras materias pienso que es la carga académica y no se sienten muy seguros a trabajar por si solos".

Asesora 4: "pues como $40 \%$ de la calificación se da por medio de actividades realizadas ya sea en su casa o aquí con nosotros lo que yo considero que indica mucho la reprobación de estos alumnos es precisamente el no cubrir las actividades y no tanto la asistencia insisto por que la asistencia no tiene ningún valor lo que tiene valor son las actividades y si el muchacho por alguna u otra razón no las realiza o no las entrega pues pierde ahí 40 puntos de calificación y eso es lo que hace que reprueben".

\subsection{Apoyo para preparación del examen de UO}

El Centro de Idiomas no otorgó apoyo formal, únicamente proporcionó información sobre fechas y horas de aplicación del examen. De manera informal, una persona recibió información de un asesor del CAA. Al respecto, cabe mencionar que ni las Facultades ni el Centro de Idiomas o CAA están obligados a dar apoyo académico (cursos o asesorías) a estudiantes que presentarán examen de UO debido a que ya cursaron dos veces la EE. En ocasiones se ofrece apoyo tutorial por medio de Programas Académicos de Formación 
Integral (PAFI) pero pocos estudiantes asisten a las sesiones y, a menudo, sólo toman dos o tres asesorías y dejan de asistir.

\subsection{Preparación para el examen de UO}

El estudiantado entrevistado afirmó haber estudiado por su cuenta con algunos materiales que generalmente se emplean en clases o en el CAA. Nadie asistió a un curso o recibió apoyo académico. El 50\% consideró que se preparó bien para aprobar el examen; el resto opinó que no se preparó lo suficiente.

\subsection{Comentarios finales de las personas entrevistadas}

Para concluir con la entrevista, se les pidió expresar algún comentario. La mayoría coincidió en que es necesario que se les proporcione más información acerca de la EE y las modalidades en que se puede cursar. También reconocieron que el incumplimiento y la falta de organización para con la EE trae como consecuencia la reprobación, por lo que aconsejaron al estudiantado ser más responsables y comprometidos. A continuación se muestran algunas citas de sus comentarios:

Entrevistado 2: "Lo principal que sean responsables cumplan con todas las actividades que le dediquen más tiempo a inglés por que aprender un idioma es muy complicado pero no difícil".

Entrevistado 7: "Me gustaría que dieran más información sobre las modalidades para cursar inglés y fechas de inscripción. En la plática de Conoce tu Universidad les dieron información pero faltaron fechas. En el SEA los alumnos debemos investigar por nuestra cuenta la información que necesitamos saber, [...] "hay que estudiar más porque es necesario".

Entrevistado 9: "Que le tomen la importancia que tiene la materia, que no la vayan dejando".

\subsection{Comentarios finales de las asesoras}

Las asesoras realizaron una fuerte crítica a las personas tutoras de cada programa educativo quienes deben proporcionar información correcta sus estudiantes ya que esto a menudo no sucede. Las asesoras enfatizaron la importancia de una tutoría efectiva para garantizar el éxito estudiantil y consideraron que las Facultades deben incluir a los CAAs y al 
Centro de Idiomas en sus actividades de información a estudiantes de nuevo ingreso. Aquí sus comentarios:

Asesora 3: "Sugiero que los maestros de las Facultad que son tutores ojala y se acercaran aquí y vinieran y supieran exactamente cómo se maneja inglés, porque les dicen a su alumnos y se les da información que muchas veces no es cierto, entonces creo que ahí es una parte importante que debería ser solucionada para que los estudiantes obtuvieran mejores resultados y tomaran mejores decisiones".

Asesora 1: "Lo que generalmente nos dicen, es que en ingles reprueban mucho, pero hemos llegado a la conclusión de que no es así, más bien hay mucha deserción de estudiantes durante el semestre, o incluso alumnos que se inscriben y jamás llegan al autoacceso, entonces ese alumno que se inscribió allá abajo que administrativamente si está inscrito pues ni siquiera llego al autoacceso, ni siquiera a veces saben que están inscritos en muchos casos, entonces hemos llegado a la conclusión de que no es tanto que reprueben si no a la deserción de los alumnos".

\section{Conclusiones}

Este estudio permitió tener un panorama más real y exacto de la situación de la reprobación en estudiantes de la EE Inglés I. Se logró obtener información valiosa proveniente de los principales participantes: estudiantes y personal académico directamente involucrado en este proceso.

De esta manera, se puede saber ahora que la mayoría de estudiantes cursan y acreditan la EE Inglés I en el tercer semestre de su PE aunque una minoría lo pospone hasta el quinto o sexto semestre.

A pesar del programa "Conoce tu Universidad", la información proporcionada a estudiantes de nuevo ingreso no es suficiente. Como consecuencia, no siempre son las estudiantes y los estudiantes sino el personal administrativo de sus facultades quien decide el momento y la modalidad en que se cursará la EE. En el caso de jóvenes que tienen oportunidad de elegir, a menudo optan por la modalidad autónoma ya que, erróneamente, piensan que el trabajo es poco y que la asistencia no es obligatoria. Esto sucede porque ignoran en qué consiste el sistema autónomo y las responsabilidades que conlleva.

En la segunda inscripción, la modalidad presencial fue la más popular. Sin embargo, a pesar de que es un sistema que ya es familiar, volvieron a reprobar.

Entre las diversas causas de reprobación identificadas destacan las siguientes: el número de EE's cursadas al mismo tiempo, la falta de interés, la ineficiente organización del 
tiempo, la dificultad para adaptarse al sistema autónomo y desconocimiento de los procesos administrativos. Estas causas coincidieron con las señaladas por el personal académico que participó en las entrevistas. Cabe mencionar que Reyes identificó causas similares de reprobación: "problemas económicos y familiares; la falta de hábitos y técnicas de estudio apropiadas; [...]" la falta de información previa a su elección..." (2002, p. 22).

\section{Propuestas}

Identificar a estudiantes con bajo desempeño escolar desde la primera inscripción y crear una red con las tutoras y los tutores para realizar acciones que prevengan la no acreditación.

Diseñar un taller dirigido a las personas tutoras de la región que les permita conocer las modalidades en que se pueden cursar las EEs de inglés. Con esto se busca que estén en mejores posibilidades de asesorar a sus tutoradas/os.

Diseñar e implementar un taller de "aprender a aprender" dirigido a estudiantes de nuevo ingreso que los familiarice con el sistema de aprendizaje autónomo o autoaprendizaje y con los criterios de evaluación establecidos por los CAAs de la región. López Segrera apoya el concepto de que el profesorado debe promover la "afición al estudio y los hábitos mentales que incentiven el autoaprendizaje (aprender a aprender)" (2008, p. 284) no sólo para inglés sino para la educación superior en general.

Se espera que este estudio posibilite la realización de futuras investigaciones en las cuales se diseñen programas de intervención que consigan disminuir los índices de reprobación en el alumnado en las EEs de inglés del AFBG y, por consiguiente, los conflictos que derivan de ella.

De igual modo, para investigaciones futuras sería conveniente correlacionar el desempeño estudiantil en la EE de inglés en relación con la situación en otras EEs.

\section{Referencias}

Aldana Franco, Rosario, Mora Barradas, Carlos Alberto, Ricaño Herrera, Francisco, Álvarez Sánchez Ervin, López Velázquez, Andrés, Navarro Piedra, José, Solórzano Hernández, Rodolfo, Ramírez Ramírez, Alfredo, García Araujo, María, Hernández Flores, Mario Franciso y Rosas, Miguel Eduardo. (20 de noviembre, 2012). Identificación de las Causas de Reprobación en la Facultad de Ingeniería Mecánica Eléctrica Región Xalapa de La Universidad Veracruzana. Divergencias.com.mx. Recuperado de http://www.divergencias.com.mx/index.php?option=com content\&view=article\&id=223:i dentificacion-de-las-causas-de-reprobacion-en-la-facultad-de-ingenieria-mecanica- 
electrica-region-xalapa-de-la-universidad-veracruzana\&catid=115:cienciassociales\&Itemid $=363$

Beltrán Casanova, Jenny. (2005). El Modelo Educativo Integral y Flexible de la Universidad Veracruzana. Revista de Investigación Educativa, (1). Instituto de Investigaciones en Educación, Universidad Veracruzana. Recuperado de: http://www.redalyc.org/pdf/2831/283121715005.pdf

Brown, H. Douglas. (2007). Principles of Language Learning and Teaching (5th ed.). White Plains, N.Y.: Pearson Education.

Cabrera Sánchez, Claudia, Romano Rodríguez, Carmen y Valenzuela Ojeda, Gloria. (2006). Causas que provocan la reprobación de los estudiantes de la licenciatura en Lingüística y Literatura Hispánica de la BUAP. Graffylia: Revista de la Facultad de Filosofía $y \quad$ Letras, (6), 143-149. Recuperado de http://www.filosofia.buap.mx/Graffylia/6/143.pdf

Creswell, John W. (1994). El procedimiento cualitativo. En Diseño de investigación. Aproximaciones cualitativas y cuantitativas (pp. 143-171). Thousand Oaks: Sage.

Hernández Sampieri, Roberto, Fernández Collado, Carlos y Baptista Lucio, Pilar. (2010) Metodología de la Investigación (5ª . ed.). D.F. México.: McGraw-Hill.

López Segrera, Francisco. (2008). Tendencias de la Educación Superior en el Mundo y en América latina $y$ del Caribe. Recuperado de http://www.ses.unam.mx/curs02014/pdf/LopezSegrera2008.pdf

Luna Martínez, Martha, Barradas Alarcón, María Esther y Balderrama Trápaga, Jorge A. (2012). Depresión en estudiantes con rezago en Inglés I y II, Universidad Veracruzana. Revista Iberoamericana para la Investigación y el Desarrollo Educativo. Recuperado de http://www.ride.org.mx/version9-10-11-12/index.php/RIDE/article/viewFile/59/58

Martinez Maldonado, Ma. de la Luz, Vivaldo Lima, Javier, Navarro Padilla María Goretti, González de la Fuente, María Virginia y Jerónimo Montes, José Antonio. (1998). Análisis Multirreferencial del Fenómeno de la Reprobación en Estudiantes Universitarios Mexicanos. Psicología Escolar y Educativa 2(2), 161-174.

México, Instituto Nacional de Estadística y Geografía (INEGI). (2010). Censo de población y vivienda 2010. Recuperado de http://www.inegi.org.mx/est/contenidos/proyectos/ccpv/cpv2010/

México, Universidad Veracruzana. (2008). Legislación Universitaria, Estatuto de los Alumnos. Xalapa: UV. Recuperado de http://www.uv.mx/legislacion/files/2012/12/estatutodelosalumnos2008.pdf

Rama Vitale, Claudio. (2006). La tercera reforma de la educación superior en América Latina y el Caribe: masificación, regulaciones e internacionalización", Revista Educación y Pedagogía, 18(46), 13-24. Recuperado de http://aprendeenlinea.udea.edu.co/revistas/index.php/revistaeyp/article/viewFile/6875/6 $\underline{292}$ 
Reyes Cruz, María del Rosario. (2002). La Reprobación en Lengua Inglesa, un primer acercamiento. Anuario de la División de Estudios Internacionales y Humanidades. Universidad de Quintana Roo. Recuperado de http://biblioteca.cogcyt.gob.mx/bvic/Captura/upload/LA-REPROBACION-EN-LENGUAINGLESA-UN-PRIMER-ACERCAMIENTO.pdf

Rodríguez Sotomayor, Juan Alberto. (1999). Investigaciones desconcentradas sobre índices y causas de reprobación, bajo rendimiento y deserción. Recuperado de http://www.galeon.com/escuela/INVESTIGACION.html\#CONTEXTO.

Yerena Aguilar, Clara Elena. (2000) Área de Formación Básica General del Nuevo Modelo Educativo (Folleto). Xalapa: Universidad Veracruzana. 


\section{Anexos}

\section{Anexo 1: Guía de entrevista para estudiantes}

Edad: Sexo: Carrera:

Facultad:

\section{PRIMERA OPORTUNIDAD}

¿En qué semestre cursaste tu primera oportunidad de Inglés I?

¿Qué información te proporcionó tu secretario académico y/o tutor antes de inscribirte a la EE de inglés en tu primera inscripción?

Si no se te proporcionó información, ¿trataste de buscar información por tu cuenta? ¿Dónde?

¿En qué modalidad cursaste la experiencia educativa de inglés 1 en la primera oportunidad? ¿Por qué te inscribiste en esta modalidad?

¿Qué pasó en tu primera inscripción a esta EE? ¿A qué atribuyes el que hayas reprobado o perdido esta primera oportunidad? Factores que influyeron para que reprobaras:

¿Cuántas materias estudiabas al momento de llevar inglés como primera inscripción?

¿Había alguna actividad que interfiriera con tus clases de inglés (horario/ tareas o proyectos de otras EEs/trabajo):

\section{SEGUNDA OPORTUNIDAD}

\section{¿Cuánto tiempo pasó entre la primera y segunda oportunidad?}

¿En qué semestre cursaste tu segunda oportunidad?

¿En qué modalidad te inscribiste en la segunda oportunidad? ¿Por qué elegiste esa modalidad?

¿Qué pasó en la segunda oportunidad? ¿A qué atribuyes a que no haya sido exitoso tu paso por esta EE? Factores que influyeron para que reprobaras:

¿Cuántas materias estudiabas al momento de llevar inglés en tu segunda inscripción?

¿Había alguna actividad que interfiriera con tus clases de inglés (horario/ tareas o proyectos de otras EEs/trabajo)? 


\section{ÚLTIMA OPORTUNIDAD}

¿Cuándo solicitaste tu examen de UO a la Coordinación del Centro de idiomas? ¿Al principio o fin de semestre?

¿Recibiste apoyo en tu facultad o en el centro de idiomas para prepararte para tu examen?

¿Te preparaste para tu examen? ¿Cómo?

$\square$ estudiaste por tu cuenta $\square$ - contrataste un maestro $\square$ - asistías a otra escuela de inglés

口- asistías de oyente a un grupo del $\mathrm{Cl}$ 口- otro

¿Piensas que te preparaste lo suficiente para aprobar examen de UO?

Resultados del examen

Escrito: $\quad$ Oral: $\_$Resultado: $\square$ Aprobado $\square$ Reprobado

Comentarios finales del entrevistado:

\section{Anexo 2: Guía de entrevista para asesores del CAA}

Desde su punto de vista, ¿que saben los usuarios de nuevo ingreso acerca del CAA y de la modalidad autónoma? ¿Qué información traen?

¿Qué comentarios ha escuchado acerca de las razones por las que los usuarios han elegido esta modalidad?

Describa como familiarizan a los usuarios nuevos con la manera en que deben trabajar en el CAA. Mencione quienes están a cargo de esto.

¿En qué consiste la modalidad autónoma que se imparte aquí? ¿Cuáles son las actividades específicas que los usuarios deben realizar y cómo?

¿De qué manera evalúan a los usuarios?

¿Cuáles piensa que son las causas principales por las que reprueban la EE?

¿Qué habilidades específicas necesitas los estudiantes para aprobar la EE en la modalidad autónoma?

¿Ha apoyado de alguna manera a alumnos que han presentado examen de UO? Describa

¿Desea añadir algún comentario?

\section{Anexo 3: Guía de entrevista para profesores de grupos presenciales}

Desde su punto de vista, ¿que saben los alumnos de nuevo ingreso acerca de la EE Inglés I y II MEIF? ¿Qué información traen?

¿Qué comentarios ha escuchado acerca de las razones por las que los alumnos han elegido esta modalidad?

¿En qué consiste la modalidad presencial que imparte? ¿Cuáles son las actividades específicas que los estudiantes deben realizar y cómo?

¿De qué manera evalúa a los estudiantes?

¿Cuáles piensa que son las causas principales por las que reprueban la EE?

¿Qué habilidades específicas necesitas los estudiantes para aprobar la EE en la modalidad presencial?

¿Ha apoyado de alguna manera a alumnos que presentarán examen de UO? Describa

¿Desea añadir algún comentario? 